\title{
C-C Motif Chemokine 5 Attenuates Angiotensin II-Dependent Kidney Injury by Limiting Renal Macrophage Infiltration
}

Nathan P. Rudemiller, ${ }^{* \dagger}$ Mehul B. Patel, ${ }^{* \dagger}$ Jian-dong Zhang, ${ }^{* \dagger}$ Alexander D. Jeffs, ${ }^{* \dagger}$ Norah S. Karlovich, ${ }^{* \dagger}$ Robert Griffiths, ${ }^{* \dagger}$ Matthew J. Kan, ${ }^{\ddagger}$ Anne F. Buckley, Michael D. Gunn, ${ }^{\ddagger}$ and Steven D. Crowley ${ }^{\star \dagger}$

From the Divisions of Nephrology* and Cardiology, ${ }^{\ddagger}$ Department of Medicine, and the Department of Pathology, ${ }^{\S}$ Duke University Medical Center, Durham; and the Durham VA Medical Center, ${ }^{\dagger}$ Durham, North Carolina

Accepted for publication

July 19, 2016.

Address correspondence to Steven D. Crowley, M.D., MSRB2 Rm 2018, Box 103015, Durham, NC 27710. E-mail: steven.d. crowley@duke.edu.

\begin{abstract}
Inappropriate activation of the renin angiotensin system (RAS) is a key contributor to the pathogenesis of essential hypertension. During RAS activation, infiltration of immune cells into the kidney exacerbates hypertension and renal injury. However, the mechanisms underpinning the accumulation of mononuclear cells in the kidney after RAS stimulation remain unclear. C-C motif chemokine 5 (CCL5) drives recruitment of macrophages and T lymphocytes into injured tissues, and we have found that RAS activation induces CCL5 expression in the kidney during the pathogenesis of hypertension and renal fibrosis. We therefore evaluated the contribution of CCL5 to renal damage and fibrosis in hypertensive and normotensive models of RAS stimulation. Surprisingly, during angiotensin II-induced hypertension, CCL5-deficient (knockout, K0) mice exhibited markedly augmented kidney damage, macrophage infiltration, and expression of proinflammatory macrophage cytokines compared with wild-type controls. When subjected to the normotensive unilateral ureteral obstruction model of endogenous RAS activation, CCL5 K0 mice similarly developed more severe renal fibrosis and greater accumulation of macrophages in the kidney, congruent with enhanced renal expression of the macrophage chemokine CCL2. In turn, pharmacologic inhibition of CCL2 abrogated the differences between CCL5 K0 and wildtype mice in kidney fibrosis and macrophage infiltration after unilateral ureteral obstruction. These data indicate that CCL5 paradoxically limits macrophage accumulation in the injured kidney during RAS activation by constraining the proinflammatory actions of CCL2. (Am J Pathol 2016, 186: 2846-2856; http://dx.doi.org/10.1016/j.ajpath.2016.07.015)
\end{abstract}

Elevated blood pressure is a significant risk factor for cardiovascular disease and chronic kidney disease (CKD). The rising prevalence of hypertension and $\mathrm{CKD}$ around the world highlights the need to further understand the underlying mechanisms involved in their pathogenesis. The cause of essential hypertension is unknown, but the efficacy of medicines that inhibit the renin angiotensin system (RAS) in lowering blood pressure indicates that the RAS is inappropriately activated in most patients with human hypertension. ${ }^{1}$ RAS-dependent hypertension leads to progressive CKD, manifested pathologically as kidney fibrosis that predicts organ failure. RAS-mediated kidney fibrosis can also arise in the absence of hypertension, ${ }^{2,3}$ but the mechanisms underpinning RAS-dependent fibrogenesis require further elucidation. Because CKD affects roughly 28 million Americans, novel therapeutic targets must be identified to reduce the risk of cardiovascular and renal complications in CKD patients that do not respond to current treatments.

A common finding in RAS-dependent hypertension and CKD is the presence of inflammatory cells in the kidney. ${ }^{4,5}$

Supported by NIH grants DK087893 and HL128355 (S.D.C.); Veterans Health Administration, Office of Research and Development, Biomedical Laboratory Research and Development grant BX000893 (S.D.C.); Edna and Fred L. Mandel Center for Hypertension and Atherosclerosis Research; grant-in-aid Fellowship from the American Heart Association; and Duke O’Brien Center for Kidney Research.

Disclosures: None declared. 
Moreover, emerging experimental data suggest that inhibiting immune system activation can reduce blood pressure and slow the progression of CKD. ${ }^{6,7}$ Macrophages and T lymphocytes accumulate around the renal vasculature and throughout the interstitium of the kidney in hypertension and during the evolution of kidney fibrosis. These infiltrating cells generate a milieu of proinflammatory and prohypertensive molecules that exacerbate tissue injury. ${ }^{8,9}$ Recent attempts to treat human metabolic and kidney diseases with novel agents that modulate mononuclear cell migration ${ }^{10}$ increase the urgency of clarifying the complex and overlapping mechanisms that govern immune cell chemoattraction during tissue damage and fibrosis.

A diverse set of chemokines coordinate the movement of immune cells from the circulation into damaged tissues, ${ }^{11}$ and a clearer understanding of how these trafficking molecules recruit inflammatory cells into the injured kidney should guide the development of novel therapies to alleviate RAS-dependent renal damage and fibrosis. C-C motif chemokine 5 (CCL5) is one of several secreted chemokines that attracts leukocytes, predominately monocytes and $\mathrm{T}$ cells, to areas of inflammation. ${ }^{12}$ CCL5 binds multiple $\mathrm{G}$ protein-coupled chemokine receptors, including $\mathrm{C}-\mathrm{C}$ chemokine receptor type (CCR)3, CCR4, and CCR5. ${ }^{13-15}$ We have previously reported that Ccl5 expression is up-regulated in the hypertensive kidney and have described exaggerated macrophage accumulation in the kidney during RAS-dependent hypertension and fibrosis. ${ }^{16} \mathrm{We}$ therefore posited that CCL5 contributes to hypertensive kidney damage and renal fibrosis. However, in the present experiments, we find that CCL5 protects the kidney during RAS activation by paradoxically limiting the CCL2-dependent recruitment of macrophages into the kidney interstitium.

\section{Materials and Methods}

All mice were housed and bred in the animal facilities at the Durham Veterans' Affairs Medical Center according to NIH guidelines. ${ }^{17}$ All animal experiments were approved by the Durham Veterans' Affairs Medical Center Institutional Animal Care and Use Committee.

\section{Mice}

All mice were bred for more than six generations onto the $129 / \mathrm{SvEv}$ background to enhance susceptibility to kidney damage. CCL5 heterozygous mice were used as experimental breeders, yielding CCL5-deficient (CCL5 KO) and wild-type (WT) littermates for experiments. Eight- to 12week-old mice were used for our studies.

\section{Chronic Ang II Infusion Model of Hypertension and Kidney Injury}

Mice underwent right nephrectomy to render the left kidney more susceptible to damage. One week after uninephrectomy, pressure transmitters (PA-C10; Data Science
International, St. Paul, MN) were surgically implanted to measure intra-arterial blood pressures by radiotelemetry in conscious, unrestrained animals. Allowing 7 days for restoration of diurnal rhythms, baseline blood pressures were recorded for 3 days. Osmotic minipumps (model 2004; Alzet, Cupertino, CA) were then implanted subcutaneously to infuse angiotensin (Ang) II (1000 ng/kg/min; A9525; Sigma-Aldrich, St. Louis, MO) continuously for 28 days. On day 25 , the mice were placed in metabolic cages, and urine was collected for 24 hours. Urinary concentrations of albumin were measured in individual samples using a specific enzyme-linked immunosorbent assay for mouse albumin (Exocell, Philadelphia, PA). Creatinine concentrations were measured with a picric acid-based method using a kit (Exocell). Albumin excretion is expressed as microgram per milligram of creatinine. On day 28 , hearts and kidneys were harvested, weighed, and analyzed as described below.

\section{UUO Model of Renal Fibrosis}

Unilateral ureteral obstruction (UUO) was performed as described previously. ${ }^{18}$ Some animals were treated with propagermanium $(8 \mathrm{mg} / \mathrm{kg}$ per day) or vehicle by oral gavage beginning on the day of UUO. Briefly, mice were anesthetized with isoflurane, and the left ureter was ligated 3 to $5 \mathrm{~mm}$ below its origin. Seven days after ligation, mice were euthanized, and the obstructed and nonobstructed kidneys were harvested for analysis.

\section{RNA Isolation and PCR Analysis}

Total RNA was isolated from cells or tissue using the RNeasy Mini Kit (Qiagen, Valencia, CA). cDNA was synthesized using the SuperScript II First-Strand Synthesis kit (Invitrogen, Carlsbad, CA). Real-time PCR was performed on an ABI 7900HT using TaqMan probes (Applied Biosystems, Foster City, CA) for Col1a1, Serpine1 (Pai-1), Tgfb1, Lcn2 (Ngal), Il1b, Tnf, Ccl1, Ccl3, Ccl4, Ccr1, Ccr5, and Ccl2.

\section{Renal Histology and Immunohistochemistry}

Transverse or sagittal portions of the kidney were fixed in $10 \%$ formalin (Sigma-Aldrich) overnight. Kidney portions were paraffin-embedded and sectioned $(5 \mu \mathrm{m})$. Sections were stained with Masson trichrome or picrosirius red for detection of fibrosis. To visualize the infiltration of macrophages and $\mathrm{T}$ lymphocytes, sections were stained with anti-F4/80 (no. MCA497G; Serotec, Oxford, United Kingdom) and anti-CD3 (clone SP7; Thermo Fisher Scientific, Waltham, MA), respectively. Interstitial fibrosis and tubular injury were scored by a pathologist masked to experimental conditions using a semiquantitative scoring system as follows: 0 , none; 1 , minimal; 2 , mild; 3, moderate; and 4, moderately severe, as described. ${ }^{19}$ For glomerular injury, the percentage of glomeruli on each section (1 section/animal) exhibiting both glomerulosclerosis and epithelial reactivity were tabulated. For collagen deposition and 
macrophage scoring, $\times 20$ field images spanning the entire cortex and outer medulla were photographed and used for analysis with color-image-analysis software (ImageJ software version 1.50; NIH, Bethesda, MD). Percentages of positive stains within the fields were averaged for each mouse and then for each group. For the hypertension model in which renal macrophage accumulation was less intense, percentage of staining was separated into quintiles to enhance the sensitivity of analysis as described. ${ }^{20}$ Perivascular T-cell infiltrates were scored in a blinded fashion (M.B.P.) according to a previously established method and reported as T cells per vessel. ${ }^{21}$

\section{Kidney Flow Cytometry}

Mice were deeply anesthetized, the lower vena cava was pierced, and $3 \mathrm{~mL}$ phosphate-buffered saline was flushed through the left ventricle. The hypertensive or obstructed kidney was collected and decapsulated. The kidney was minced in a petri dish and transferred to a $\mathrm{C}$ tube (Miltenyi Biotec, Auburn, CA) containing $5 \mathrm{~mL}$ RPMI- 1640 with $1 \mathrm{mg} /$ $\mathrm{mL}$ collagenase IV (Gibco, Carlsbad, CA) and $10 \mathrm{mg} / \mathrm{mL}$ DNase (Sigma-Aldrich). After homogenization with the GentleMACS (Miltenyi Biotec), the kidney homogenate was incubated for 30 minutes at $37^{\circ} \mathrm{C}$. The resulting cell suspension was filtered through $70-$ and $40-\mu$ cell strainers and washed twice with wash buffer (Dulbecco's phosphatebuffered saline containing $2 \%$ fetal bovine serum and $2 \mathrm{mmol} /$ L EDTA). The cells were treated with FC Block (BioLegend, San Diego, CA) and incubated with CD11b-fluorescein isothiocyanate, CD45-Brilliant Violet 510, lymphocyte antigen 6 complex (Ly6C)-phycoerythrin-cyanine 7, lymphocyte antigen 6 complex locus G (Ly6G)-Brilliant Violent 421 (all BioLegend), CD4-phosphatidylethanolamine, and Nearinfrared dead cell indicator (Life Technologies, Carlsbad, CA) for 30 minutes at 4 degrees. Cells were washed and fixed with Fix/Perm buffer (BD Biosciences, San Jose, CA). Flow cytometry was performed on a FACSCanto II (BD Biosciences) and data were analyzed with FlowJo software version 10.2 (Tree Star, Inc., Ashland, OR).

\section{Statistical Analysis}

All values are expressed as means \pm SEM. Comparison between and within groups was performed with two-tailed unpaired and paired Student's $t$-tests, respectively. Comparison among groups was performed with one-way analysis of variance or a $\chi^{2}$ test.

\section{Results}

CCL5 Limits Urinary Albumin Excretion via a Blood Pressure-Independent Mechanism

To determine the role of CCL5 in the hypertensive response to RAS activation, we subjected uninephrectomized WT and CCL5 KO mice to 4 weeks of chronic Ang II infusion and measured blood pressures by radiotelemetry. At baseline, blood pressures were similar in the WT and CCL5 KO cohorts. Both groups mounted a robust hypertensive response to Ang II, but no divergence in blood pressure emerged between the groups during the Ang II infusion period (Figure 1A). Consistent with their similar blood pressures, the WT and CCL5 KO animals developed the same degree of cardiac hypertrophy after 4 weeks of hypertension (Figure 1B). However, despite these similar blood pressures, Ang II-infused CCL5 KO mice had significantly greater urinary albumin excretion than WT mice (Figure 1C), suggesting that CCL5 deficiency may permit damage to the kidney glomerulus in the setting of hypertension through a blood pressure-independent mechanism.

\section{CCL5 Deficiency Augments Ang II-Induced Kidney Damage}

To determine whether the exaggerated albuminuria in the Ang II-infused CCL5 KO cohort reflected more severe renal injury, we examined renal pathology in the experimental groups after 4 weeks of Ang II-induced hypertension or saline control. Kidneys from the normotensive saline-infused controls did not exhibit glomerular or interstitial damage (Figure 2 and Figure 3). Compared with Ang II-infused WT animals, the Ang II-treated CCL5 KO animals had worse glomerular injury marked by glomerulosclerosis $(7.3 \% \pm 2.2 \%$ versus $11.8 \% \pm 2.4 \%)$ and epithelial cell reactivity $(10.4 \% \pm 2.4 \%$ versus $15.7 \% \pm 1.3 \%$ ) (Figure 2 ). The extent of fibrosis indicates the degree of damage and predicts functional failure in all organs, and the degree of interstitial fibrosis was markedly increased in the Ang IIinfused CCL5 KO mice compared with WT mice (Figure 3).

To evaluate whether CCL5 deficiency congruently altered gene expression programs for injury and fibrosis in the kidney during RAS activation, we measured mRNA levels for several inflammatory mediators and markers after 4 weeks of hypertension. Neutrophil gelatinase-associated lipocalin (LCN2, NGAL) is a sensitive marker and mediator of renal damage. ${ }^{22}$ Consistent with the exaggerated injury in the CCL5 KO cohort, these animals had a striking up-regulation of renal Lcn2 (Ngal) expression compared with control animals (Figure 4A). Congruent with the augmented renal fibrosis in the CCL5 KO group, expression levels of collagen 1 (Col1a1) were increased more than twofold over those in their WT counterparts (Figure 4B) as were the expressions of the key fibrosis mediators Serpine1 (Pai1) and Tgfb1 (Figure 4C). Thus, despite the known actions of CCL5 to recruit inflammatory cells into injured tissues, CCL5 deficiency augmented kidney damage and scar formation in our hypertension model.

\section{Exaggerated Accumulation of Macrophages in the CCL5-Deficient Kidney during Hypertension}

CCL5 influences macrophage trafficking, and macrophages are key regulators of tissue fibrosis. ${ }^{23}$ Therefore, to determine whether altered macrophage infiltration into the kidney 


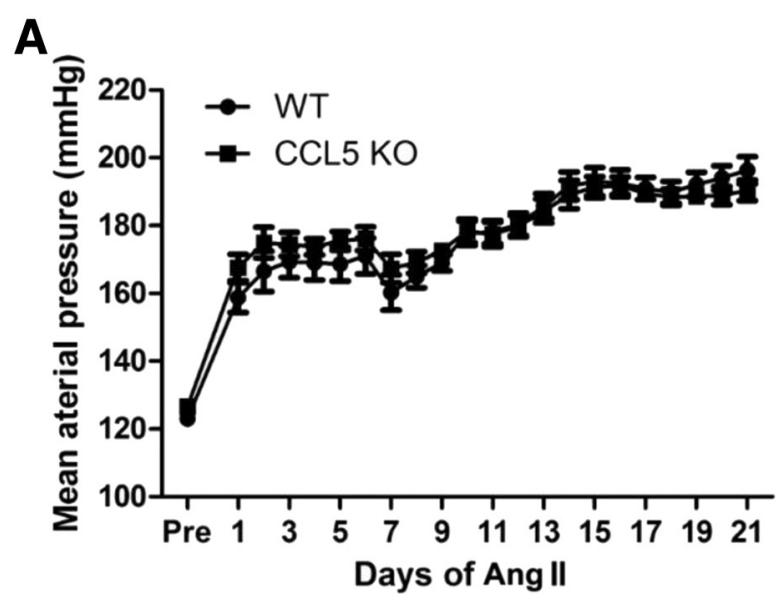

B

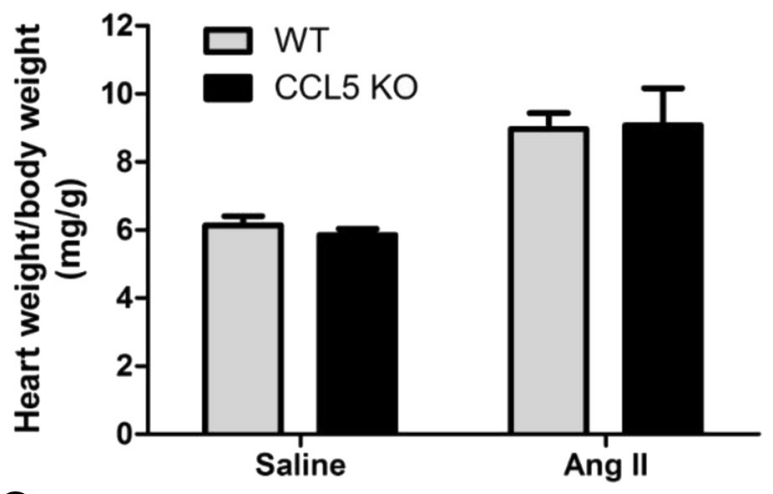

C

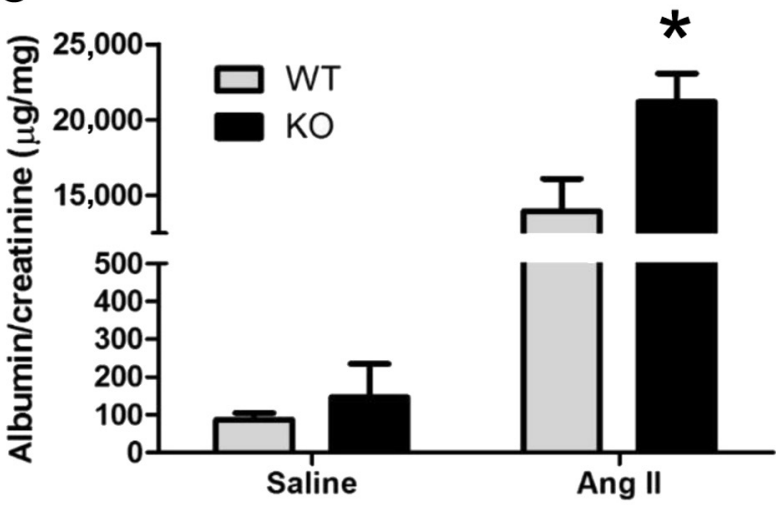

Figure 1 CCL5-deficient mice have a preserved chronic hypertensive response to Ang II. A: MAPs of WT and CCL5 K0 mice were measured by radiotelemetry before and during Ang II-dependent hypertension $\mathbf{B}$ and C: Heart weight-to-body weight (B) and albumin-to-creatinine ratios (C) were determined after 4 weeks of chronic saline or Ang II infusion in WT and CCL5 K0 mice. $n=22$ mice per group. ${ }^{*} P<0.05$ versus WT. Ang, angiotensin; CCL5, C-C motif chemokine 5; KO, knockout; MAP, mean arterial pressure; WT, wild-type.

could account for the amplified renal injury in CCL5 KO animals, we quantitated $\mathrm{F} 4 / 80^{+}$macrophage accumulation in the kidney after 4 weeks of Ang II with a scoring system we have previously optimized for counting renal $\mathrm{T}$ cells. ${ }^{20}$ Although kidney sections from both groups showed robust staining for macrophages dispersed throughout the renal interstitium, the degree of macrophage accumulation was significantly enhanced in the CCL5 KO cohort $(P<0.0001)$ (Figure 5, A-C). Similarly, blinded histologic scoring (M.B.P.) revealed a trend toward enhanced accumulation of $\mathrm{T}$ lymphocytes around the renal blood vessels in the hypertensive CCL5 KO animals $(P<0.07)$ (Supplemental Figure S1), and fluorescent cell sorting isolated significantly more $\mathrm{CD}^{+}{ }^{+} \mathrm{T}$ cells from these CCL5 KO kidneys than from WT hypertensive controls $(12.3 \pm 1.8$ versus $5.7 \pm 0.8$ per 10,000 kidney cells; $P<0.03$ ).

Macrophages elaborate proinflammatory cytokines that mediate tissue injury and fibrosis, including IL-1 $\beta$ and tumor necrosis factor (TNF)- $\alpha .^{24,25}$ After 4 weeks of hypertension, mRNA levels of Il1b and Tnf in the CCL5 KO kidney were 2.5 -fold and threefold, respectively, of WT values, respectively, whereas IL-6 was similarly expressed in the two groups (Figure 5D). These data suggest that CCL5 mitigates kidney fibrosis during RAS activation by suppressing the renal accumulation of inflammatory macrophages that generate profibrotic cytokines.

To determine whether up-regulation of the receptors for CCL5 or alternative ligands for these receptors in the CCL5 KO kidneys could account for the enhanced renal accumulation of mononuclear cells in the CCL5 cohort after 4 weeks of hypertension, we measured renal mRNA expression for these receptors and ligands in our experimental animals (Figure 5E). CCR5 (Ccr5), the prototypical receptor for CCL5, was similarly expressed in the two groups. CCR1 (Ccr1), an alternative receptor for CCL5, was downregulated in the CCL5 KOs. Among the alternative ligands for these receptors, CCL1 (Ccl1) was not detectable in the hypertensive kidneys, CCL3 (Ccl3) was similarly expressed in the groups, and CCL4 (Ccl4) was down-regulated in the CCL5 KO cohort. Because none of these CCL5 receptors or alternative CCL5 ligands was up-regulated in the CCL5 KOs, we measured gene expression for the monocyte and T-cell chemokine CCL2 (Ccl2) and, in this case, detected higher levels of $\mathrm{Ccl} 2$ expression in the CCL5 KO kidneys compared with WT kidneys $(1.2 \% \pm 0.15 \%$ versus $0.82 \% \pm 0.25 \%$ ), suggesting that CCL5 deficiency permits up-regulation of CCL2.

\section{CCL5 Attenuates Renal Scar Formation after Ureteral Obstruction}

RAS activation makes a critical contribution to kidney fibrosis after UUO. ${ }^{26}$ Thus, to determine whether CCL5 regulates RAS-dependent renal fibrosis in the absence of blood pressure elevation, we subjected CCL5 KO and WT mice to the UUO model and evaluated the deposition of collagen fibrils after 7 days as measured by staining for picrosirius red (Figure 6, A-C). The contralateral, unobstructed kidneys showed minimal fibrosis in both groups. By contrast, the obstructed kidneys from the CCL5 KO mice had significantly more fibrosis than WT controls, 
A

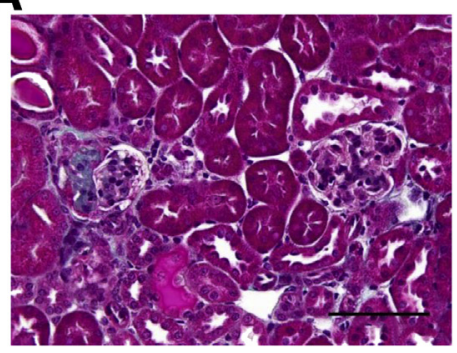

B

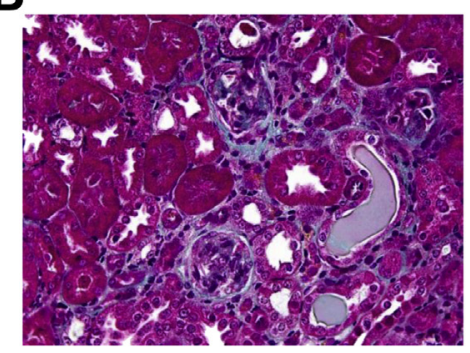

C

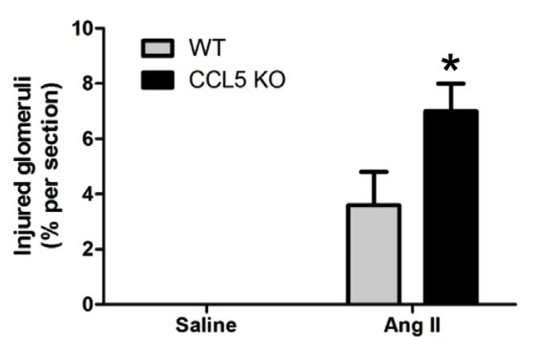

Figure 2 CCL5 mitigates Ang II-mediated glomerular injury. After 28 days of chronic saline or Ang II infusion, kidney sections of WT and CCL5 K0 mice were blindly assessed (A.F.B.) for glomerular injury. A and B: Representative kidney sections from Ang II-infused WT (A) and CCL5 KO (B) mice. Injured glomeruli were scored based on the combined levels of glomerulosclerosis and epithelial cell reactivity (C). CCL5 KO mice had augmented glomerular injury compared with WT controls. ${ }^{*} P<0.05$ versus WT. Scale bar $=100 \mu \mathrm{m}(\mathbf{A}$ and B). Ang, angiotensin; AU, arbitrary units; CCL5, C-C motif chemokine 5; K0, knockout; WT, wild-type.

corroborating our findings from the hypertensive model of RAS activation.

\section{CCL5 Deficiency Prompts Additional Accumulation of Macrophages in the Obstructed Kidney}

Because UUO is a more robust model of renal macrophage infiltration than the hypertensive model of RAS activation, we quantitated $\mathrm{F} 4 / 80^{+}$macrophage accumulation in the UUO kidney at 7 days with morphometric scoring. Here, again, obstructed kidneys from the CCL5 KO group revealed more intense macrophage staining than WT controls (Figure 6, D-F). UUO led to more than a 100 -fold induction of mRNA for the chemokine CCL2 in the WT kidney $(122.2 \pm 27.0$ versus $1.0 \pm 0.2$ arbitrary units; $P<0.001)$. Nevertheless, consistent with the enhanced macrophage accumulation in the CCL5 KO obstructed kidneys, expression of $C c l 2$ was markedly up-regulated in the CCL5 KO obstructed kidneys compared with WT controls (Figure 6G). However, this enhanced expression of CCL2 was not due to its up-regulation in the infiltrating CCL5 KO macrophages because Ccl2 mRNA expression was similar in the infiltrating macrophages isolated from the obstructed WT and CCL5 KO kidneys $(1.0 \pm 0.1$ versus $1.0 \pm 0.2$ arbitrary units).

\section{CCL5 Mitigates the Accumulation of Proinflammatory Ly6C $\mathrm{C}^{\text {hi }}$ Monocytes/Macrophages in the Obstructed Kidney}

Robust expression of the Ly6C on circulating monocytes marks a proinflammatory monocyte population that is released from the bone marrow and recruited to injured tissue via a CCR2-dependent mechanism. ${ }^{27,28}$ These cells mediate inflammation by releasing the proinflammatory cytokines IL$1 \beta$ and TNF. ${ }^{29}$ Seven days after UUO, cells were isolated from the obstructed kidneys of CCL5 KO and WT mice, and the level of expression of Ly6C on renal myeloid cells was determined by flow cytometric analysis. Ly6 $\mathrm{C}^{\text {hi }}$ expression was augmented on the CCL5 KO compared with the WT myeloid cells (Figure 7), yielding more CD $11 b^{+}$Ly $_{6 G^{-}}{\text {Ly } 6 C^{\text {hi }}}$ inflammatory monocytes in the CCL5 KO group (176 \pm 11 versus $142 \pm 9$ per 1000 renal leukocytes; $P=0.037)$. Thus, CCL5 attenuates the recruitment of proinflammatory monocytes into the kidney during RAS activation.

\section{CCL2 Up-Regulation Worsens RAS-Dependent Fibrosis in the Absence of CCL5}

From these findings, we posited that, in the absence of CCL5, up-regulation of CCL2 leads to exaggerated
A

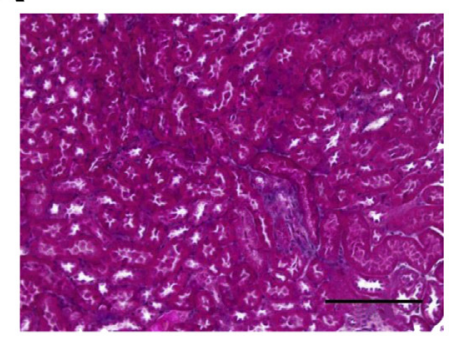

B

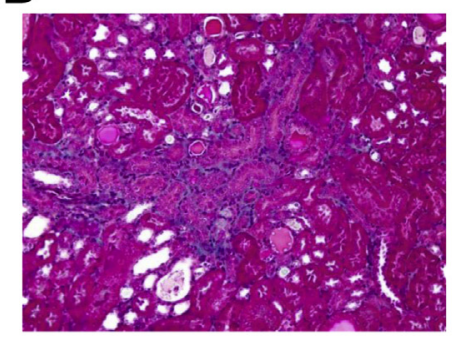

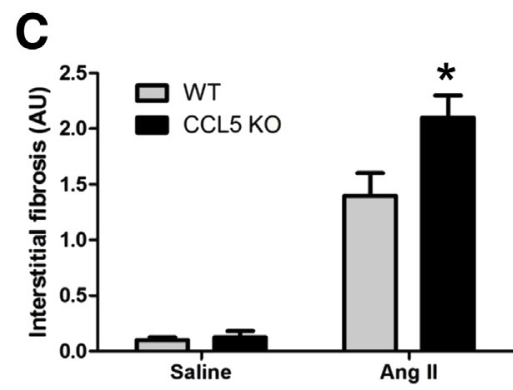

Figure 3 CCL5 protects from renal interstitial damage by RAS activation. After 4 weeks of saline control or Ang II-induced hypertension, sections of WT and CCL5 K0 kidneys were blindly analyzed (A.F.B.) using a semiquantitative scoring method of interstitial fibrosis ranging from no injury (0) to severe fibrosis (4). A and B: Representative kidney sections from Ang II-infused WT (A) and CCL5 K0 (B) mice. C: Fibrosis scores. Ang II-infused CCL5 K0 mice were significantly more susceptible to kidney fibrosis. ${ }^{*} P<0.05$ versus WT. Scale bar $=100 \mu \mathrm{m}(\mathbf{A}$ and B). Ang, angiotensin; AU, arbitrary units; CCL5, C-C motif chemokine 5; $\mathrm{K} 0$, knockout; RAS, renin angiotensin system; WT, wild-type. 

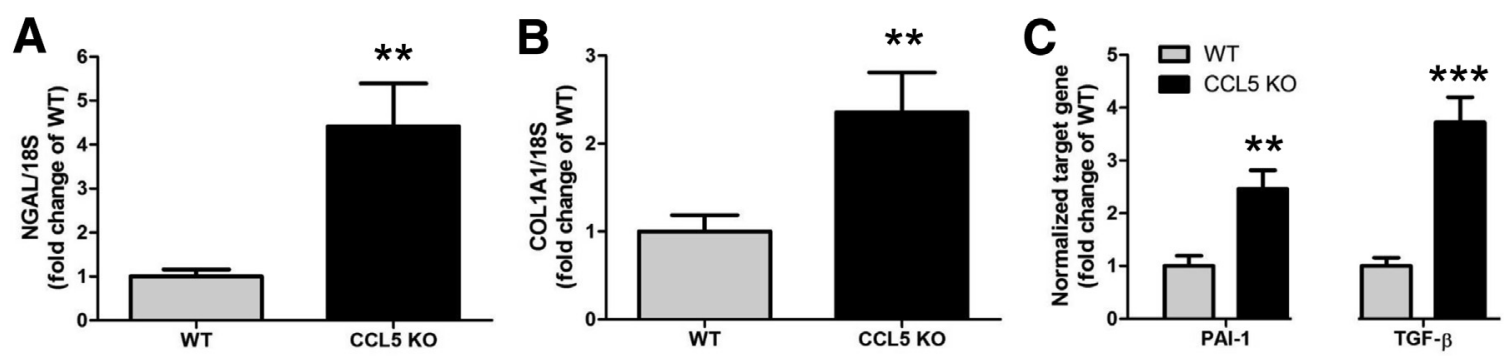

Figure 4 mRNA expression of markers and mediators of hypertensive kidney injury. Whole kidney mRNA expression of Lcn2 (A), Col1a1 (B), Serpine1 (Pai1), and Tgfb1 (C) was determined in WT and CCL5 KO mice after 28 days of chronic Ang II infusion. Expression of these injury markers was elevated in CCL5 KO mice compared with WT controls. ${ }^{* *} P<0.01$ and ${ }^{* * *} P<0.001$ versus WT. Ang, angiotensin; CCL5, C-C motif chemokine 5; K0, knockout; WT, wild-type.

macrophage accumulation in the kidney with a consequent increase in the severity of renal fibrosis. To directly test this possibility, we treated WT and CCL5 KO animals after UUO with the CCL2 inhibitor propagermanium $(8 \mathrm{mg} / \mathrm{kg}$ per day). CCL2 inhibition reduced levels of fibrosis in both cohorts and abrogated the differences in fibrosis between the WT and CCL5 KO cohorts (Figure 8, A-C). Moreover, CCL2 blockade congruently reduced renal macrophage infiltration down to similar levels in the WT and CCL5 KO groups (Figure 8, D-F). Thus, antagonizing CCL2 ameliorates the increased kidney macrophage accumulation and renal scar formation permitted by CCL5 deficiency during RAS activation.

\section{Discussion}

CCL5 is one of several chemokines that directs circulating immune cells to sites of inflammation via binding of $G$ protein-coupled chemokine receptors. ${ }^{30}$ CCL5 is generated by many cell types, including epithelial and endothelial cells in the kidney, ${ }^{31-34}$ particularly during activation of RAS, ${ }^{35,36}$ and interacts with CCR1, CCR3, and CCR5. Studies have determined that immune cell populations, especially $\mathrm{T}$ cells and macrophages, infiltrate the kidney and amplify injury and fibrosis in hypertension driven by activation of RAS, ${ }^{37,38}$ and CCL5 is up-regulated in the kidney in the Ang II-dependent hypertension and UUO

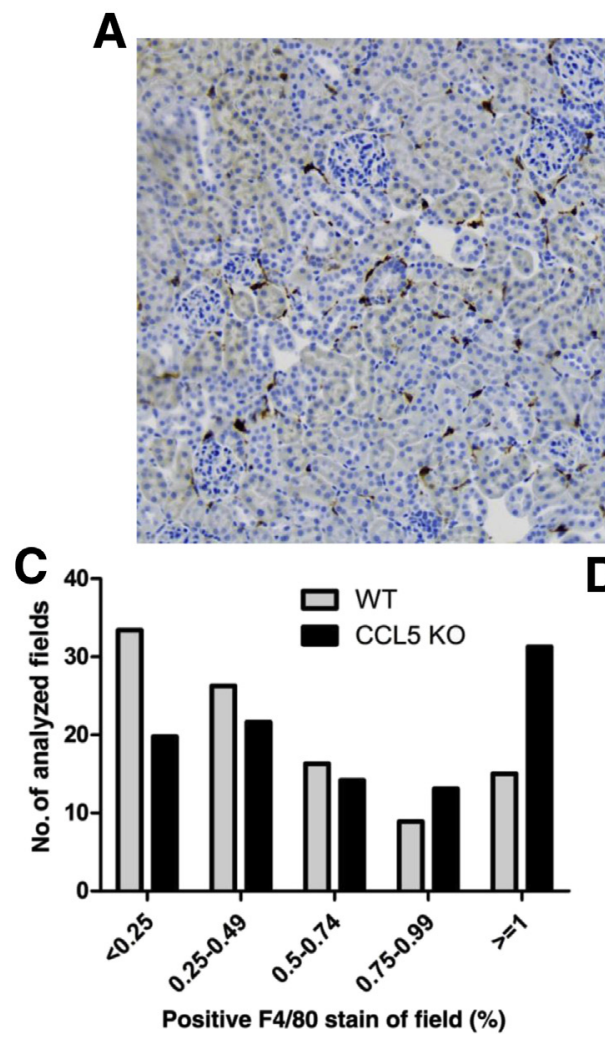

\section{B}

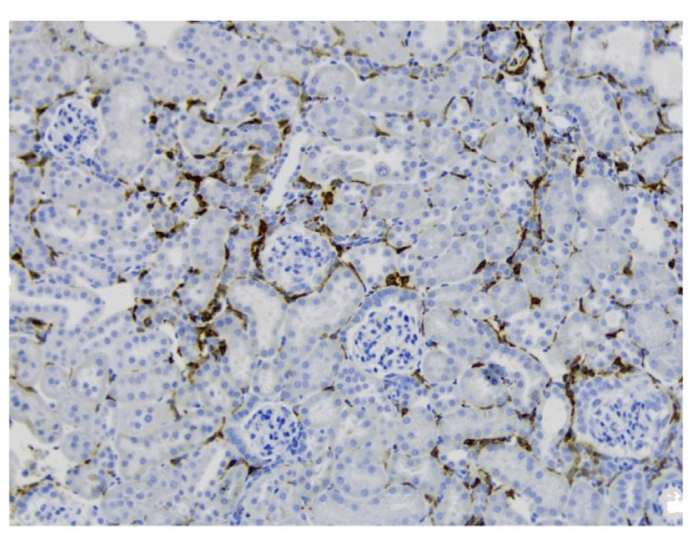

D

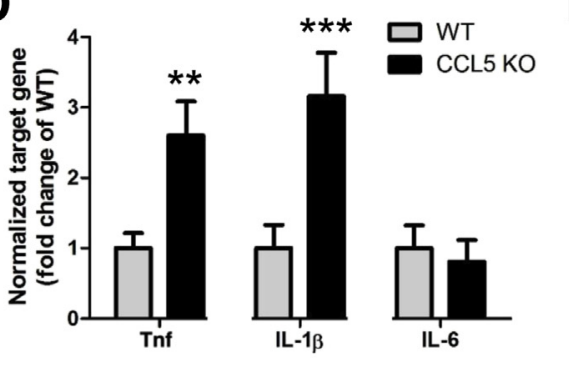

E

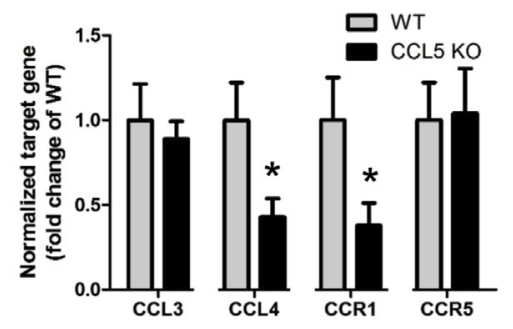

Figure 5 CCL5 deficiency permits augmented macrophage infiltration and inflammation in the hypertensive kidney. A and B: WT (A) and CCL5 K0 (B) kidney sections were stained for the macrophage marker F4/80 after 28 days of Ang II. C: The density of macrophage staining was digitally scored into quintiles and subjected to $\chi^{2}$ analysis $(P<0.0001)$. D and E: mRNA levels of the proinflammatory mediators Tnf, Il $1 \mathrm{~b}$, and II6 (D) and the alternative CCL5 ligands C $\mathrm{Cl} 3$ and Ccl4 and the CCL5 receptors Ccr1 and CCr5 (E) were measured in WT and CCL5 K0 kidneys. ${ }^{*} P<0.05,{ }^{* *} P<0.01$, and ${ }^{* * *} P<0.001$ versus WT. Scale bar $=100 \mu \mathrm{m}(\mathbf{A}$ and B). Ang, angiotensin; CCL5, C-C motif chemokine; CCR, C-C chemokine receptor; K0, knockout; Tnf, tumor necrosis factor; WT, wild-type. 


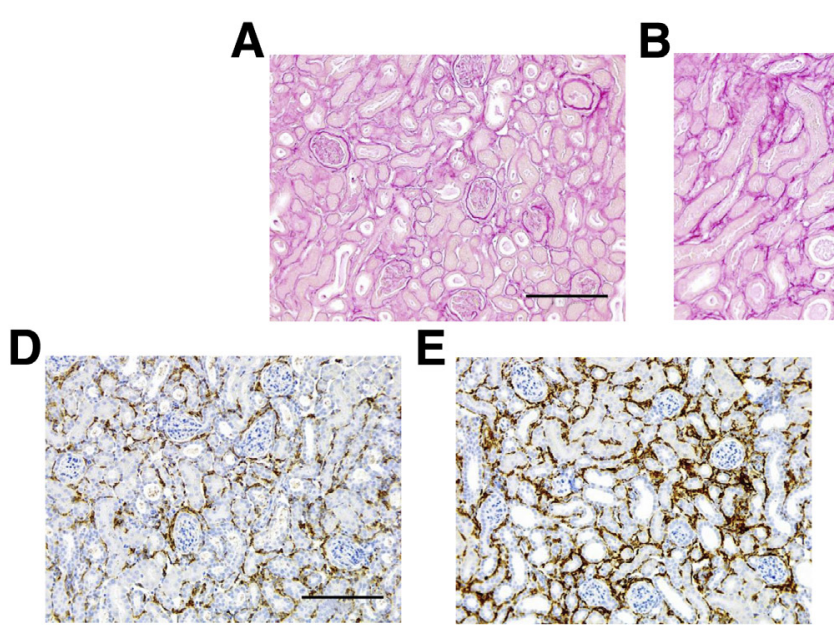

$\mathbf{F}$

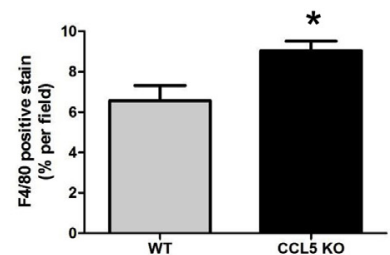

C

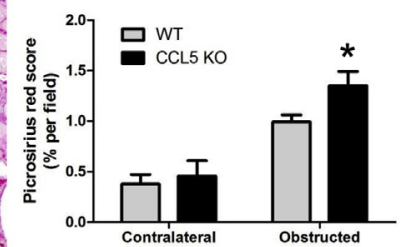

G

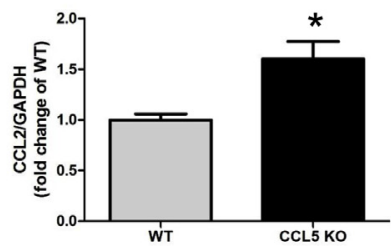

Figure 6 CCL5 deficiency exaggerates fibrosis and infiltration of macrophages in the obstructed kidney. A and B: Seven days after UU0, sections of WT (A) and CCL5 KO (B) kidneys were stained with picrosirius red to visualize collagen fibrils. C: The percentage of picrosirius red stain per section was digitally quantified in obstructed and contralateral, unobstructed kidneys. D-F: Sections of WT (D) and CCL5 K0 (E) obstructed kidneys were stained with anti-F4/80, and the percentage of positive stain per section was computed (F). G: mRNA expression of Ccl2, a strong chemoattractant for macrophages, was up-regulated in obstructed CCL5 K0 compared with WT kidneys. Scale bars $=100 \mu \mathrm{m}\left(\mathbf{A}, \mathbf{B}, \mathbf{D}\right.$, and E). ${ }^{*} P<0.05$ versus WT. CCL5, C-C motif chemokine 5; K0, knockout; UUO, unilateral ureteral obstruction; WT, wild-type.

models of RAS activation. ${ }^{16,19,39}$ We therefore posited that CCL5 recruits mononuclear cells into the kidney to propagate blood pressure elevation and/or renal damage and fibrosis.
The present studies revealed that CCL5 deficiency does not modulate blood pressure at baseline or during chronic Ang II infusion. Consistent with our studies, Krebs et $\mathrm{al}^{40}$ found that deleting CCR5, the receptor for CCL5, had no

A

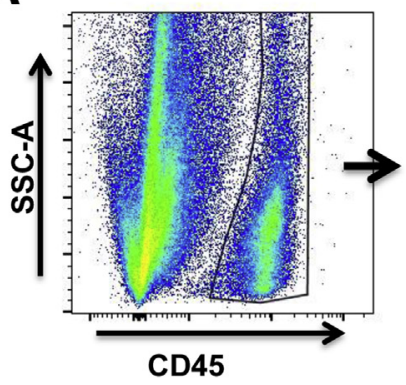

B

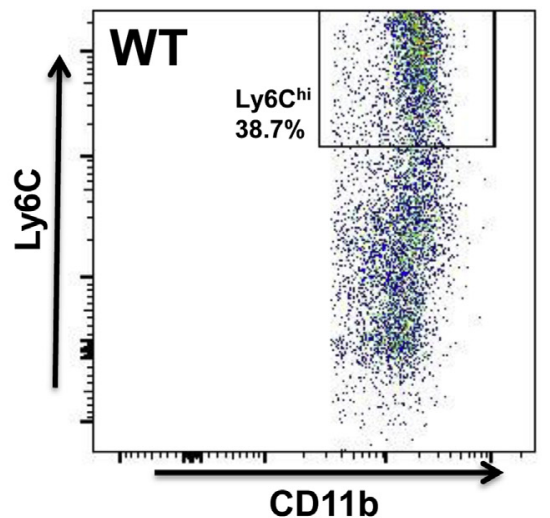

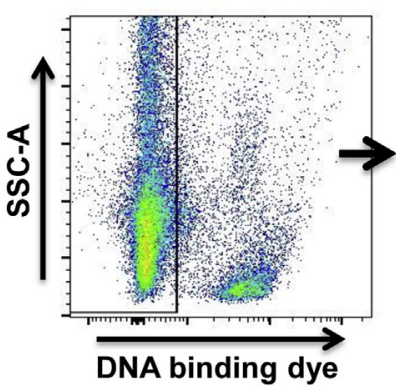

DNA binding dye

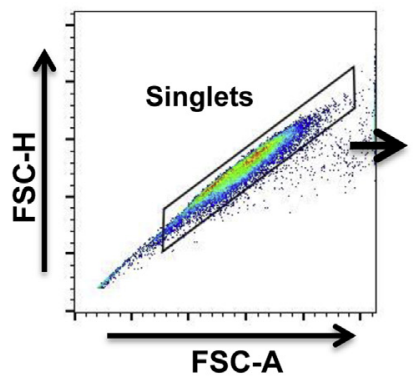

FSC-A

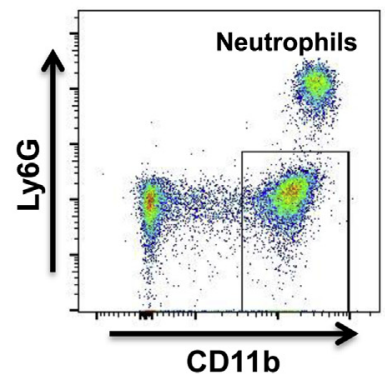

C

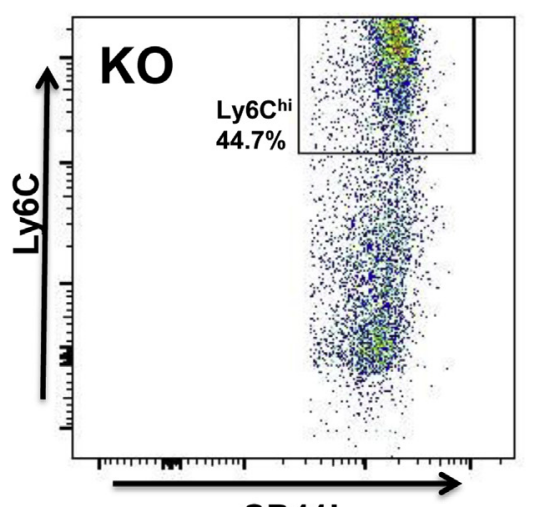

CD11b

Figure 7 CCL5 limits the infiltration of inflammatory Ly6 $\mathrm{C}^{\text {hi }}$ myeloid cells into the obstructed kidney. Seven days after UU0, renal myeloid cells were evaluated via flow cytometry for their expression of Ly6C. A: The gating strategy for analyzing viable myeloid cells. B and C: Within the myeloid cell population, Ly6 $\mathrm{C}^{\mathrm{hi}}$ expression was significantly enhanced in the CCL5 K0 obstructed kidney compared with WT. ${ }^{*} P<0.01$ versus WT. CCL5, C-C motif chemokine 5 ; FSC, forward scatter; K0, knockout; Ly6C, lymphocyte antigen 6 complex; SSC, side scatter; UU0, unilateral ureteral obstruction; WT, wild-type. 

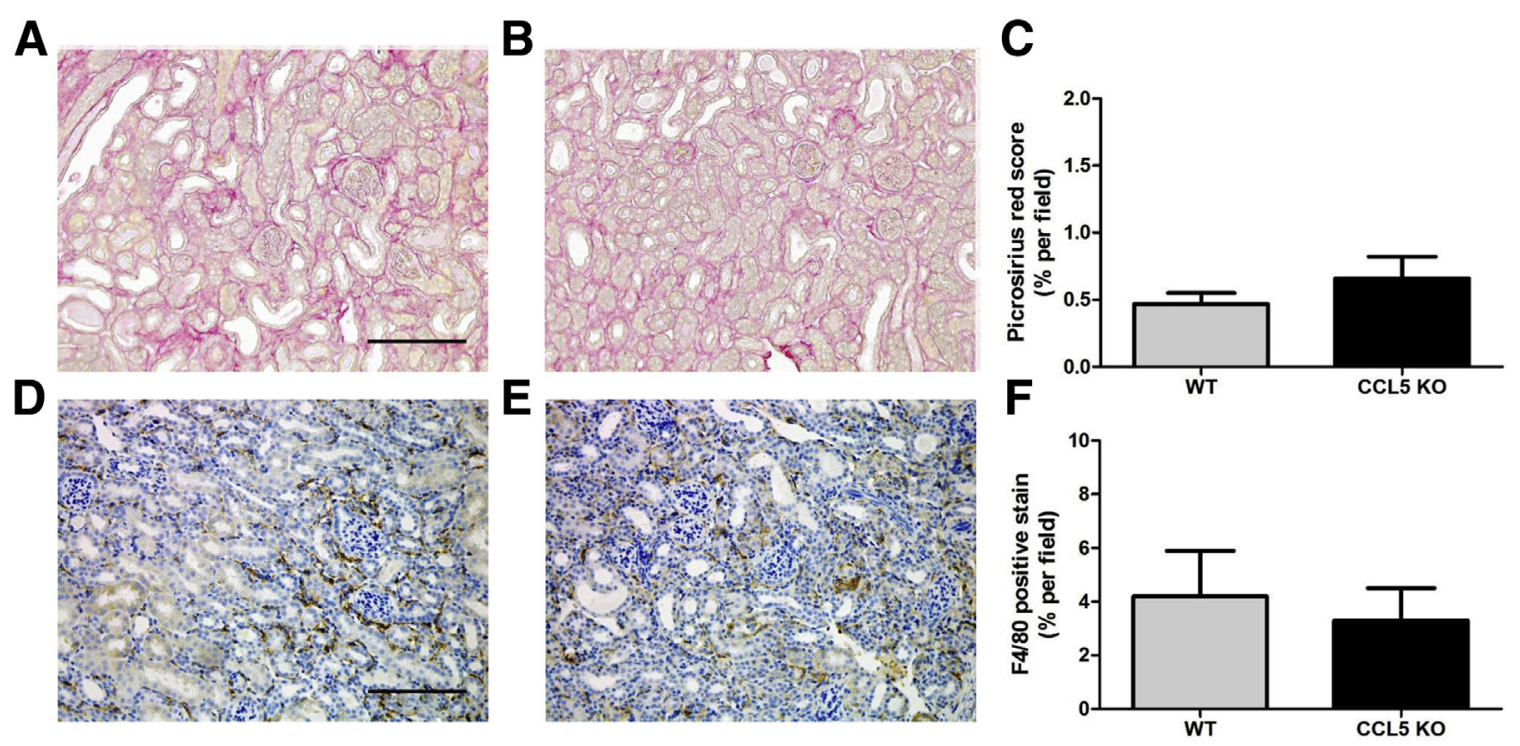

Figure 8 CCL2 antagonism reduces UU0-induced fibrosis and macrophage infiltration in the CCL5 K0 kidneys to WT levels. Mice were treated with the CCL2 inhibitor propagermanium after UU0. A-C: CCL2 inhibition yields similar levels of picrosirius red staining for collagen fibrils in obstructed WT (A) and CCL5 K0 (B) kidneys, quantified by morphometric analysis (C). D-F: CCL2 antagonist reduces and renders macrophage infiltration comparable in obstructed WT (D) and CCL5 K0 (E) kidneys, quantified in panel F. Scale bars $=100 \mu \mathrm{m}(\mathbf{A}, \mathbf{B}, \mathbf{D}$, and E). CCL2, C-C motif chemokine 2; CCL5, C-C motif chemokine 5; K0, knockout; UUO, unilateral ureteral obstruction; WT, wild-type.

impact on the hypertensive response in a combined DOCAsalt/Ang II infusion model. ${ }^{40}$ Because deleting the receptor for CCL2, another key macrophage and T-cell chemokine, also did not influence the hypertensive response during RAS activation, ${ }^{41}$ our studies would suggest that these chemokines modulate renal injury through blood pressureindependent mechanisms. However, pharmacologic doses of CCL5 in the bloodstream or central nervous system can influence blood pressure homeostasis, ${ }^{42,43}$ indicating that a threshold dose of CCL5 may be required to mediate hemodynamic effects.

Although CCL5 did not influence blood pressure in our experiments, CCL5 deficiency led to exaggerated hypertensive renal damage during RAS stimulation, particularly within the kidney glomerulus. These results contradicted our initial hypothesis that CCL5 would exacerbate renal injury by promoting accumulation of inflammatory cells in the kidney. Nevertheless, some studies of immune-mediated glomerulonephritis have supported a role for CCL5 to ameliorate disease in the kidney glomerulus. For example, CCL5 blockade worsened crescentic glomerulonephritis in one case by increasing the activation of macrophages in the kidney. ${ }^{44,45}$ Moreover, deficiency of the CCL5 receptor, CCR5, augmented crescentic glomerulonephritis and murine lupus nephritis. ${ }^{46,47}$ These findings are congruent with the enhanced glomerulosclerosis and albuminuria recorded in our CCL5deficient cohort during Ang II-dependent hypertension and may accrue from the previously reported induction of CCL5 in the glomerular endothelium during RAS activation. ${ }^{35}$ However, the protective actions of CCL5 appear to be specific to the kidney because CCL5 has been reported to promote vascular inflammation and endothelial dysfunction, and CCL5-deficient bone marrow chimeras had blunted atherosclerosis in the apolipoprotein E-deficient preclinical model. ${ }^{48,49}$

Given the chemotactic functions of CCL5, we were surprised to discover that CCL5 deficiency augments macrophage accumulation in the hypertensive kidney. Consistent with a role for these macrophages to instigate the exaggerated renal damage in the CCL5-deficient animals, their kidneys showed marked up-regulation of two key macrophage cytokines, TNF and IL-1 $\beta$, that we and others have implicated in the pathogenesis of hypertensive kidney damage driven by RAS activation. ${ }^{6,21,50}$ For example, TNF is directly toxic to the kidney glomerulus. ${ }^{51}$ Thus, CCL5 appears to limit the extent of renal damage during RAS-mediated hypertension by suppressing the infiltration into the kidney of inflammatory macrophages and the injurious cytokines they elaborate.

Because macrophages are key regulators of kidney fibrosis, we evaluated the extent of kidney scar formation in our hypertensive animals and found that CCL5 suppresses mediators of fibrosis, including Tgfb1 and Serpine1 (Pai1), and matrix deposition in the injured kidney. However, the degree of renal macrophage infiltration and fibrosis is relatively mild in the Ang II hypertension model. We therefore confirmed our findings in the normotensive UUO model, which features robust macrophage accumulation in the kidney, marked kidney scar formation, ${ }^{52}$ and endogenous RAS activation. ${ }^{26}$ Here, again, CCL5 deficiency permitted enhanced macrophage infiltration into the injured kidney and an increase in the severity of renal fibrosis. Because CCL5 has been reported to potentiate scar formation in the liver, ${ }^{53}$ the actions of CCL5 to protect the kidney from fibrosis also appear to be tissue specific. 
The accumulation of tissue macrophages during injury depends on the infiltration of circulating monocytes that can present diverse phenotypes. Therefore, we sought to delineate the monocyte populations in the obstructed kidney of CCL5 KO and WT mice by measuring their level of Ly6C expression. Ly6C is a membrane surface protein, and its robust expression designates a particular subset of proinflammatory, bone marrow-derived monocytes. These Ly6 $\mathrm{C}^{\text {hi }}$ monocytes, although absent in the healthy kidney, are recruited into the injured kidney ${ }^{54,55}$ and harbor a propensity for differentiation to M1 macrophages, which produce high levels of TNF and IL- $1 \beta .{ }^{56}$ Consistent with the exaggerated injury in the CCL5 KO kidneys during RAS activation, we found a significantly higher proportion of Ly6C ${ }^{\text {hi }}$ proinflammatory myeloid cells in the CCL5 KO cohort. Recruitment of Ly6C ${ }^{\text {hi }}$ monocytes is mediated by the macrophage chemokine CCL2, which was up-regulated in the CCL5 KO kidneys. ${ }^{57,58}$ Moreover, like CCL5, CCL2 is up-regulated in damaged endothelium ${ }^{59}$ and makes a critical contribution to the pathogenesis of kidney fibrosis. ${ }^{52}$ We posited, therefore, that a compensatory induction of CCL2 drove the increase in inflammatory macrophage accumulation in the CCL5 KO kidney, leading to exaggerated renal damage and fibrosis. Consistent with this hypothesis, pharmacologic inhibition of CCL2 reduced macrophage infiltration and fibrosis in both experimental groups and abrogated the differences between the groups in both of these parameters. By contrast, we could not detect up-regulation in the CCL5 KOs of a receptor for CCL5 or an alternative CCR5 ligand that would otherwise explain the enhanced mononuclear cell accumulation in the injured kidneys of the CCL5 KO animals.

\section{Conclusions}

In summary, despite reported functions of CCL5 to recruit mononuclear cells into injured tissues, we find that CCL5 attenuates macrophage infiltration into the kidney during RAS activation and thereby mitigates the extent of RAS-dependent renal damage and fibrosis. To resolve this paradox, we have established that the absence of CCL5 permits augmented induction of the macrophage chemokine CCL2. Accordingly, blockade of CCL2 prevents the enhanced renal macrophage infiltration and fibrosis accruing from CCL5 deficiency. These studies illustrate the complex interrelations between mononuclear chemokines and the potential danger of manipulating their signaling pathways in kidney disease without a complete understanding of their overlapping functions in disease pathogenesis. As agents that target CCL2 signaling are beginning to achieve some therapeutic success in patients with metabolic syndrome and CKD,${ }^{10}$ clarifying the interactions between CCL5 and CCL2 will become increasingly critical to guide the emerging translation of chemokine modulators for the treatment of CKD and other fibrogenic disorders.

\section{Supplemental Data}

Supplemental material for this article can be found at http://dx.doi.org/10.1016/j.ajpath.2016.07.015.

\section{References}

1. Dahlof B, Devereux RB, Kjeldsen SE, Julius S, Beevers G, de Faire U, Fyhrquist F, Ibsen H, Kristiansson K, LederballePedersen O, Lindholm LH, Nieminen MS, Omvik P, Oparil S, Wedel H; LIFE Study Group: Cardiovascular morbidity and mortality in the Losartan Intervention For Endpoint reduction in hypertension study (LIFE): a randomised trial against atenolol. Lancet 2002, 359: 995-1003

2. Ruiz-Ortega M, Ruperez M, Esteban V, Rodriguez-Vita J, SanchezLopez E, Carvajal G, Egido J: Angiotensin II: a key factor in the inflammatory and fibrotic response in kidney diseases. Nephrol Dial Transplant 2006, 21:16-20

3. Wolf G: Renal injury due to renin-angiotensin-aldosterone system activation of the transforming growth factor-beta pathway. Kidney Int 2006, 70:1914-1919

4. Zhang J, Crowley SD: Role of T lymphocytes in hypertension. Curr Opin Pharmacol 2015, 21:14-19

5. Imig JD, Ryan MJ: Immune and inflammatory role in renal disease. Compr Physiol 2013, 3:957-976

6. Muller DN, Shagdarsuren E, Park JK, Dechend R, Mervaala E, Hampich F, Fiebeler A, Ju X, Finckenberg P, Theuer J, Viedt C, Kreuzer J, Heidecke H, Haller H, Zenke M, Luft FC: Immunosuppressive treatment protects against angiotensin II-induced renal damage. Am J Pathol 2002, 161:1679-1693

7. Guzik TJ, Hoch NE, Brown KA, McCann LA, Rahman A, Dikalov S, Goronzy J, Weyand C, Harrison DG: Role of the T cell in the genesis of angiotensin II induced hypertension and vascular dysfunction. J Exp Med 2007, 204:2449-2460

8. Ramseyer VD, Garvin JL: Tumor necrosis factor-alpha: regulation of renal function and blood pressure. Am J Physiol Renal Physiol 2013 , 304:F1231-F1242

9. Itani HA, Harrison DG: Memories that last in hypertension. Am J Physiol Renal Physiol 2015, 308:F1197-F1199

10. de Zeeuw D, Bekker P, Henkel E, Hasslacher C, Gouni-Berthold I, Mehling H, Potarca A, Tesar V, Heerspink HJ, Schall TJ: CCX140-B Diabetic Nephropathy Study Group: The effect of CCR2 inhibitor CCX140-B on residual albuminuria in patients with type 2 diabetes and nephropathy: a randomised trial. Lancet Diabetes Endocrinol 2015, 3:687-696

11. Le Y, Zhou Y, Iribarren P, Wang J: Chemokines and chemokine receptors: their manifold roles in homeostasis and disease. Cell Mol Immunol 2004, 1:95-104

12. Schall TJ, Bacon K, Toy KJ, Goeddel DV: Selective attraction of monocytes and $\mathrm{T}$ lymphocytes of the memory phenotype by cytokine RANTES. Nature 1990, 347:669-671

13. Power CA, Meyer A, Nemeth K, Bacon KB, Hoogewerf AJ, Proudfoot AE, Wells TN: Molecular cloning and functional expression of a novel CC chemokine receptor cDNA from a human basophilic cell line. J Biol Chem 1995, 270:19495-19500

14. Samson M, Labbe O, Mollereau C, Vassart G, Parmentier M: Molecular cloning and functional expression of a new human CC-chemokine receptor gene. Biochemistry 1996, 35:3362-3367

15. Ponath PD, Qin S, Ringler DJ, Clark-Lewis I, Wang J, Kassam N, Smith H, Shi X, Gonzalo JA, Newman W, Gutierrez-Ramos JC, Mackay CR: Cloning of the human eosinophil chemoattractant, eotaxin. Expression, receptor binding, and functional properties suggest a mechanism for the selective recruitment of eosinophils. J Clin Invest 1996, 97:604-612 
16. Zhang JD, Patel MB, Song YS, Griffiths R, Burchette J, Ruiz P, Sparks MA, Yan M, Howell DN, Gomez JA, Spurney RF, Coffman TM, Crowley SD: A novel role for type 1 angiotensin receptors on T lymphocytes to limit target organ damage in hypertension. Circ Res 2012, 110:1604-1617

17. Committee for the Update of the Guide for the Care and Use of Laboratory Animals, National Research Council: Guide for the Care and Use of Laboratory Animals: Eighth Edition. Washington, DC, National Academies Press, 2011

18. Chevalier RL, Forbes MS, Thornhill BA: Ureteral obstruction as a model of renal interstitial fibrosis and obstructive nephropathy. Kidney Int 2009, 75:1145-1152

19. Zhang JD, Patel MB, Griffiths R, Dolber PC, Ruiz P, Sparks MA, Stegbauer J, Jin H, Gomez JA, Buckley AF, Lefler WS, Chen D, Crowley SD: Type 1 angiotensin receptors on macrophages ameliorate IL-1 receptor-mediated kidney fibrosis. J Clin Invest 2014, 124: $2198-2203$

20. Crowley SD, Frey CW, Gould SK, Griffiths R, Ruiz P, Burchette JL, Howell DN, Makhanova N, Yan M, Kim HS, Tharaux PL, Coffman TM: Stimulation of lymphocyte responses by angiotensin II promotes kidney injury in hypertension. Am J Physiol Renal Physiol 2008, 295:F515-F524

21. Crowley SD, Song YS, Sprung G, Griffiths R, Sparks M, Yan M, Burchette JL, Howell DN, Lin EE, Okeiyi B, Stegbauer J, Yang Y, Tharaux PL, Ruiz P: A role for angiotensin II type 1 receptors on bone marrow-derived cells in the pathogenesis of angiotensin II-dependent hypertension. Hypertension 2010, 55:99-108

22. Viau A, El Karoui K, Laouari D, Burtin M, Nguyen C, Mori K, Pillebout E, Berger T, Mak TW, Knebelmann B, Friedlander G, Barasch J, Terzi F: Lipocalin 2 is essential for chronic kidney disease progression in mice and humans. J Clin Invest 2010, 120:4065-4076

23. Meng XM, Nikolic-Paterson DJ, Lan HY: Inflammatory processes in renal fibrosis. Nat Rev Nephrol 2014, 10:493-503

24. Lan HY, Nikolic-Paterson DJ, Zarama M, Vannice JL, Atkins RC: Suppression of experimental crescentic glomerulonephritis by the interleukin-1 receptor antagonist. Kidney Int 1993, 43:479-485

25. Lan HY, Yang N, Metz C, Mu W, Song Q, Nikolic-Paterson DJ, Bacher M, Bucala R, Atkins RC: TNF-alpha up-regulates renal MIF expression in rat crescentic glomerulonephritis. Mol Med 1997, 3: 136-144

26. Satoh M, Kashihara N, Yamasaki Y, Maruyama K, Okamoto K, Maeshima Y, Sugiyama H, Sugaya T, Murakami K, Makino H: Renal interstitial fibrosis is reduced in angiotensin II type 1a receptordeficient mice. J Am Soc Nephrol 2001, 12:317-325

27. Gordon S, Taylor PR: Monocyte and macrophage heterogeneity. Nat Rev Immunol 2005, 5:953-964

28. Karlmark KR, Weiskirchen R, Zimmermann HW, Gassler N, Ginhoux F, Weber C, Merad M, Luedde T, Trautwein C, Tacke F: Hepatic recruitment of the inflammatory Gr1+ monocyte subset upon liver injury promotes hepatic fibrosis. Hepatology 2009, 50:261-274

29. Serbina NV, Jia T, Hohl TM, Pamer EG: Monocyte-mediated defense against microbial pathogens. Annu Rev Immunol 2008, 26:421-452

30. Krensky AM, Ahn YT: Mechanisms of disease: regulation of RANTES (CCL5) in renal disease. Nat Clin Pract Nephrol 2007, 3: $164-170$

31. Zoja C, Donadelli R, Colleoni S, Figliuzzi M, Bonazzola S, Morigi M, Remuzzi G: Protein overload stimulates RANTES production by proximal tubular cells depending on NF-kappa B activation. Kidney Int 1998, 53:1608-1615

32. Kuroiwa T, Schlimgen R, Illei GG, McInnes IB, Boumpas DT: Distinct $\mathrm{T}$ cell/renal tubular epithelial cell interactions define differential chemokine production: implications for tubulointerstitial injury in chronic glomerulonephritides. J Immunol 2000, 164:3323-3329

33. Segerer S, Djafarzadeh R, Grone HJ, Weingart C, Kerjaschki D, Weber C, Kungl AJ, Regele H, Proudfoot AE, Nelson PJ: Selective binding and presentation of CCL 5 by discrete tissue microenvironments during renal inflammation. J Am Soc Nephrol 2007, 18:1835-1844
34. Deckers JG, De Haij S, van der Woude FJ, van der Kooij SW, Daha MR, van Kooten C: IL-4 and IL-13 augment cytokine- and CD40-induced RANTES production by human renal tubular epithelial cells in vitro. J Am Soc Nephrol 1998, 9:1187-1193

35. Wolf G, Ziyadeh FN, Thaiss F, Tomaszewski J, Caron RJ, Wenzel U, Zahner G, Helmchen U, Stahl RA: Angiotensin II stimulates expression of the chemokine RANTES in rat glomerular endothelial cells. Role of the angiotensin type 2 receptor. J Clin Invest 1997, 100: 1047-1058

36. Mateo T, Abu Nabah YN, Abu Taha M, Mata M, Cerda-Nicolas M, Proudfoot AE, Stahl RA, Issekutz AC, Cortijo J, Morcillo EJ, Jose PJ, Sanz MJ: Angiotensin II-induced mononuclear leukocyte interactions with arteriolar and venular endothelium are mediated by the release of different CC chemokines. J Immunol 2006, 176:5577-5586

37. Harrison DG: The immune system in hypertension. Trans Am Clin Climatol Assoc 2014, 125:130-138. discussion 138-140

38. Quiroz Y, Johnson RJ, Rodriguez-Iturbe B: The role of T cells in the pathogenesis of primary hypertension. Nephrol Dial Transplant 2012, 27(Suppl 4):iv2-iv5

39. Crisman JM, Richards LL, Valach DP, Franzoni DF, Diamond JR: Chemokine expression in the obstructed kidney. Exp Nephrol 2001, 9:241-248

40. Krebs C, Fraune C, Schmidt-Haupt R, Turner JE, Panzer U, Quang MN, Tannapfel A, Velden J, Stahl RA, Wenzel UO: CCR5 deficiency does not reduce hypertensive end-organ damage in mice. Am J Hypertens 2012, 25:479-486

41. Liao TD, Yang XP, Liu YH, Shesely EG, Cavasin MA, Kuziel WA, Pagano PJ, Carretero OA: Role of inflammation in the development of renal damage and dysfunction in angiotensin II-induced hypertension. Hypertension 2008, 52:256-263

42. Kim HY, Cha HJ, Kim HS: CCL5 upregulates IL-10 expression and partially mediates the antihypertensive effects of IL-10 in the vascular smooth muscle cells of spontaneously hypertensive rats. Hypertens Res 2015, 38:666-674

43. Gouraud SS, Waki H, Bhuiyan ME, Takagishi M, Cui H, Kohsaka A, Paton JF, Maeda M: Down-regulation of chemokine Ccl5 gene expression in the NTS of SHR may be pro-hypertensive. J Hypertens 2011, 29:732-740

44. Lloyd CM, Dorf ME, Proudfoot A, Salant DJ, Gutierrez-Ramos JC: Role of MCP-1 and RANTES in inflammation and progression to fibrosis during murine crescentic nephritis. J Leukoc Biol 1997, 62 . 676-680

45. Anders HJ, Frink M, Linde Y, Banas B, Wornle M, Cohen CD, Vielhauer V, Nelson PJ, Grone HJ, Schlondorff D: CC chemokine ligand 5/RANTES chemokine antagonists aggravate glomerulonephritis despite reduction of glomerular leukocyte infiltration. J Immunol 2003, 170:5658-5666

46. Turner JE, Paust HJ, Bennstein SB, Bramke P, Krebs C, Steinmetz OM, Velden J, Haag F, Stahl RA, Panzer U: Protective role for CCR5 in murine lupus nephritis. Am J Physiol Renal Physiol 2012, 302:F1503-F1515

47. Turner JE, Paust HJ, Steinmetz OM, Peters A, Meyer-Schwesinger C, Heymann F, Helmchen U, Fehr S, Horuk R, Wenzel U, Kurts C, Mittrucker HW, Stahl RA, Panzer U: CCR5 deficiency aggravates crescentic glomerulonephritis in mice. J Immunol 2008, 181: 6546-6556

48. Koenen RR, von Hundelshausen P, Nesmelova IV, Zernecke A, Liehn EA, Sarabi A, Kramp BK, Piccinini AM, Paludan SR, Kowalska MA, Kungl AJ, Hackeng TM, Mayo KH, Weber C: Disrupting functional interactions between platelet chemokines inhibits atherosclerosis in hyperlipidemic mice. Nat Med 2009, 15:97-103

49. Mikolajczyk TP, Nosalski R, Szczepaniak P, Budzyn K, Osmenda G, Skiba D, Sagan A, Wu J, Vinh A, Marvar PJ, Guzik B, Podolec J, Drummond G, Lob HE, Harrison DG, Guzik TJ: Role of chemokine RANTES in the regulation of perivascular inflammation, T-cell accumulation, and vascular dysfunction in hypertension. FASEB J 2016, 30:1987-1999 
50. Zhang J, Patel MB, Griffiths R, Mao A, Song YS, Karlovich NS, Sparks MA, Jin H, Wu M, Lin EE, Crowley SD: Tumor necrosis factor-alpha produced in the kidney contributes to angiotensin II-dependent hypertension. Hypertension 2014, 64:1275-1281

51. Bertani T, Abbate M, Zoja C, Corna D, Perico N, Ghezzi P, Remuzzi G: Tumor necrosis factor induces glomerular damage in the rabbit. Am J Pathol 1989, 134:419-430

52. Kitagawa K, Wada T, Furuichi K, Hashimoto H, Ishiwata $\mathrm{Y}$, Asano M, Takeya M, Kuziel WA, Matsushima K, Mukaida N, Yokoyama H: Blockade of CCR2 ameliorates progressive fibrosis in kidney. Am J Pathol 2004, 165:237-246

53. Berres ML, Koenen RR, Rueland A, Zaldivar MM, Heinrichs D, Sahin H, Schmitz P, Streetz KL, Berg T, Gassler N, Weiskirchen R, Proudfoot A, Weber C, Trautwein C, Wasmuth HE: Antagonism of the chemokine $\mathrm{Ccl} 5$ ameliorates experimental liver fibrosis in mice. $\mathrm{J}$ Clin Invest 2010, 120:4129-4140

54. Clements M, Gershenovich M, Chaber C, Campos-Rivera J, Du P, Zhang M, Ledbetter S, Zuk A: Differential Ly6C expression after renal ischemia-reperfusion identifies unique macrophage populations. J Am Soc Nephrol 2016, 27:159-170
55. Lin SL, Castano AP, Nowlin BT, Lupher ML Jr, Duffield JS: Bone marrow Ly6Chigh monocytes are selectively recruited to injured kidney and differentiate into functionally distinct populations. J Immunol 2009, 183:6733-6743

56. Auffray C, Fogg D, Garfa M, Elain G, Join-Lambert O, Kayal S, Sarnacki S, Cumano A, Lauvau G, Geissmann F: Monitoring of blood vessels and tissues by a population of monocytes with patrolling behavior. Science 2007, 317:666-670

57. Hammond MD, Taylor RA, Mullen MT, Ai Y, Aguila HL, Mack M, Kasner SE, McCullough LD, Sansing LH: CCR2+ Ly6C(hi) inflammatory monocyte recruitment exacerbates acute disability following intracerebral hemorrhage. J Neurosci 2014, 34:3901-3909

58. Tsou CL, Peters W, Si Y, Slaymaker S, Aslanian AM, Weisberg SP, Mack M, Charo IF: Critical roles for CCR2 and MCP-3 in monocyte mobilization from bone marrow and recruitment to inflammatory sites. J Clin Invest 2007, 117:902-909

59. Weber KS, Nelson PJ, Grone HJ, Weber C: Expression of CCR2 by endothelial cells: implications for MCP-1 mediated wound injury repair and in vivo inflammatory activation of endothelium. Arterioscler Thromb Vasc Biol 1999, 19:2085-2093 